\title{
Erratum to: Re-irradiation with cetuximab or cisplatin-based chemotherapy for recurrent squamous cell carcinoma of the head and neck
}

\author{
Nicolas Dornoff - Christian Weiß - Franz Rödel • \\ Jens Wagenblast · Shahram Ghanaati • \\ Atefeh Nateghian · Claus Rödel • Panagiotis Balermpas
}

Online publiziert: 4. August 2015

(C) Springer-Verlag Berlin Heidelberg 2015

\section{Erratum to:}

\section{Strahlenther Onkol 2015}

Doi:10.1007/s00066-015-0854-y

Unfortunately, in the article "Re-irradiation with cetuximab or cisplatin-based chemotherapy for recurrent squamous cell carcinoma of the head and neck", the surname of the author Atefeh Nateghian was erroneously published as the first name and vice versa.

The correct list of authors reads as follows:

Nicolas Dornoff ${ }^{1}$, Christian Weiß ${ }^{1}$, Franz Rödel ${ }^{1}$, Jens Wagenblast $^{4}$, Shahram Ghanaati ${ }^{5}$, Atefeh Nateghian ${ }^{1,2,3}$, Claus Rödel ${ }^{1,2,3}$, Panagiotis Balermpas s, $^{1,2,3}$

The online version of the original article can be found at doi:10.1007/s00066-014-0606-4

Dr. P. Balermpas $(\bowtie) \cdot$ N. Dornoff · C. Weiß · F. Rödel ·

A. Nateghian · C. Rödel

Department of Radiotherapy and Oncology,

J. W. Goethe University,

Frankfurt a. M., Germany

e-mail: panagiotis.balermpas@kgu.de

Dr. P. Balermpas · A. Nateghian · C. Rödel

German Cancer Research Center (DKFZ) Heidelberg,

Heidelberg, Germany

A. Nateghian · C. Rödel · Dr. P. Balermpas

German Cancer Consortium (DKTK) partner site: Frankfurt,

Frankfurt a. M., Germany

J. Wagenblast

Department of Otorhinolaryngology, J. W. Goethe University,

Frankfurt a. M., Germany

S. Ghanaati

Department of Maxillofacial Surgery, J. W. Goethe University,

Frankfurt a. M., Germany
${ }^{1}$ Department of Radiotherapy and Oncology, J. W. Goethe University, Frankfurt a. M., Germany

${ }^{2}$ German Cancer Research Center (DKFZ) Heidelberg, Germany

${ }^{3}$ German Cancer Consortium (DKTK) partner site: Frankfurt, Frankfurt a. M., Germany

${ }^{4}$ Department of Otorhinolaryngology, J. W. Goethe University, Frankfurt a. M., Germany

${ }^{5}$ Department of Maxillofacial Surgery, J. W. Goethe University, Frankfurt a. M., Germany

We apologize for any inconveniences caused. 
